\title{
BIOCOMBUSTIBLES LÍQUIDOS EN COLOMBIA Y SU IMPACTO EN MOTORES DE COMBUSTIÓN INTERNA. UNA REVISIÓN
}

Johana Marcela Amarís ${ }^{1}$, Diego Antonio Manrique², Julián Ernesto Jaramillo ${ }^{3 *}$

* A quien debe dirigirse la correspondencia.

\begin{abstract}
RESUMEN
En los últimos siglos, el hombre se ha visto obligado a recurrir a diversas fuentes para garantizar sus necesidades energéticas. Es por esto, que a través de los años ha desarrollado tecnologías para explotar los combustibles fósiles, sin tener en cuenta el deterioro generado en el medio ambiente. Esta explotación indiscriminada ha llevado a los recursos naturales al borde de su agotamiento, lo cual ha impulsado el estudio de alternativas energéticas "amigables" con el medio ambiente. En este sentido, los biocombustibles se presentan como una alternativa viable a los combustibles fósiles. Éste artículo está enfocado en el estudio de los biocombustibles líquidos (bioetanol y biodiesel) por medio de una revisión bibliográfica detallada con el propósito de establecer el estado actual de esta tecnología en Colombia. Además, se han estudiado los impactos que su uso genera en los motores de combustión interna, específicamente los de ciclo Otto y Diésel.
\end{abstract}

Palabras clave: Energía renovable, Biocombustibles, Bioetanol, Biodiesel, Motores.

\section{LIQUID BIOFUELS IN COLOMBIA AND ITS IMPACT ON INTERNAL COMBUSTION ENGINES. A REVIEW}

\begin{abstract}
In the last centuries, the human being has been forced to use different sources in order to guarantee his energetic demand. It is for this reason that through the time technologies to exploiting fossil fuels have been developed, without taking into account the environment deterioration. Furthermore, this indiscriminate exploitation has brought natural resources to their depletion. These factors have promoted the study of energies environmentally "friendly". In this sense, biofuels seem to be a viable alternative to fossil fuels. This article is focused on the study of liquid biofuels in Colombia (bioethanol and biodiesel) by means of a detailed bibliographic revision. It is done to establish the state of the art of this technology. Moreover, the consequences of using them in internal combustion engines, particularly on those based on Otto and Diesel principles, are assessed.
\end{abstract}

Keywords: Renewable energy, Biofuels, Bioethanol, Biodiesel, Engines.

1. Escuela de Ingeniería Mecánica, Universidad Industrial de Santander, UIS, Carrera 27 calle 9, Bucaramanga, Colombia. Email: joy.amaris@gmail.com

2. Escuela de Ingeniería Mecánica, Universidad Industrial de Santander, UIS, Carrera 27 calle 9, Bucaramanga, Colombia. Email: diegomanriquem12@gmail.com

3. Escuela de Ingeniería Mecánica, Universidad Industrial de Santander, UIS, Carrera 27 calle 9, Bucaramanga, Colombia..Email: jejarami@uis.edu.co 


\section{INTRODUCCIÓN}

Los biocombustibles han mostrado ser una alternativa energética en varios países como Brasil, Estados Unidos, Colombia, España, Alemania, Argentina, Uruguay, Chile, Francia, India, Japón, etc. No obstante, aún se encuentran en desarrollo y tienen una alta viabilidad a corto y mediano plazo. A diferencia del petróleo, el carbón y el gas natural, estos están catalogados como una forma segura de energía, puesto que su producción se origina mediante recursos renovables. Son combustibles que contribuyen a disminuir las emisiones de gases de efecto invernadero y óxidos de azufre causantes de fenómenos como la lluvia ácida.

El panorama actual revela que los biocombustibles son un recurso para mitigar el aumento e inestabilidad en los precios del petróleo, pero no representan una alternativa para eliminar dicha crisis, debido a que una sustitución total del combustible fósil por biodiesel y bioetanol, sería agrícolamente inviable. La extensión de tierra necesaria para cultivar la materia prima destinada a la producción de biocombustibles es tan grande, que pondría en riesgo y hasta podría llegar a desplazar los cultivos con fines alimenticios (Goméz, Samaniego \& Antonissen, 2008).

Es importante resaltar que la aplicación del bioetanol y del biodiesel como fuente energética se encuentra en el sector automotriz, como combustible para motores de combustión interna y motores de compresión, a diferencia de las demás fuentes de energía, que suelen tener como fin la generación de energía eléctrica.

Este trabajo está estructurado de la siguiente manera: una introducción acerca de los biocombustibles líquidos y su clasificación, los biocombustibles en Colombia, el impacto que los biocombustibles generan en los motores de combustión interna y las líneas de investigación actuales de los biocombustibles.

\section{BIOCOMBUSTIBLES LÍQUIDOS}

Los biocombustibles son básicamente alcoholes, éteres, ésteres y otros compuestos químicos producidos a partir de la biomasa. Por ejemplo, plantas herbáceas y leñosas, residuos de la agricultura y actividad forestal; y una gran cantidad de desechos industriales, como son los desperdicios de la industria alimenticia (Stratta, 2000).

Entre los biocombustibles líquidos se pueden incluir el bioetanol, el biodiesel y el biometanol, entre otros.
Siendo el bioetanol y el biodiesel los más desarrollados y utilizados en el país actualmente. El bioetanol se produce a partir de cultivos tales como caña de azúcar, maíz, remolacha, trigo y sorgo (Stratta, 2000). El biodiesel se produce a partir de semillas oleaginosas como girasol, soya; o de frutos de árboles como la palma africana, la Jatropha o el cocotero (Dufey, 2006).

La sustitución de los combustibles denominados fósiles derivados del petróleo, por otros de origen vegetal, cobra una gran importancia en nuestros días por varias razones fundamentales: i) provienen de una fuente renovable; ii) son un instrumento de lucha contra el deterioro medioambiental, y ii) son un factor de desarrollo de la agricultura e industrias derivadas y el sector automotriz y de transporte (Dufey, 2006; Instituto Interamericano de Cooperación para la Agricultura, 2005; Sanchez \& Cardona, 2006).

En (Machado, 2010) clasifican los biocombustibles de acuerdo a la materia prima utilizada y la tecnología para obtenerlos así:

- Biocombustibles de primera generación: Son aquellos productos de plantas comestibles. En la actualidad ya han alcanzado una etapa de producción comercial. Su expansión genera preocupación en términos de uso de tierras y seguridad alimentaria.

- Biocombustibles de segunda generación: También conocidos como biocombustibles lignocelulósicos, son producidos de materias primas no pertenecientes a la dieta alimentaria de los seres humanos.

- Biocombustibles de tercera generación: Son producidos a partir de materia prima modificada. Son especialmente los aceites, procedentes de las algas y el hidrógeno procedente de la biomasa. Aún se encuentran en una etapa de desarrollo.

Como ya se comentó, este artículo se centra en los dos tipos de biocombustibles más usados en los motores de combustión interna y especialmente en Colombia, bioetanol y biodiesel.

\section{A. BIOETANOL}

Es un alcohol carburante que en la primera generación se obtiene a través del proceso de fermentación de almidones y azúcares de plantas ricas en estos (caña de azúcar, remolacha, yuca, etc.) y de cereales como maíz, trigo y sorgo; materias primas comestibles.

No obstante, debido a la enorme polémica por la seguridad alimentaria a nivel mundial en relación al 
posible desabastecimiento de alimentos derivado de la producción masiva de biocombustibles, se están investigando otras fuentes, con mayor énfasis en biomasa residual de procesos industriales. Es allí donde aparecen los llamados biocombustibles de segunda generación. Estos se obtienen a partir del material lignocelulósico presente en los productos forestales tales como maderas, cáscaras, tallos, matorrales de corta rotación y pastos energéticos (Dufey, 2006; Sanchez \& Cardona, 2006). Debido a las características de la materia prima usada, esta se ha de someter a procesos de conversión termoquímica o bioquímica, para obtener un producto que pueda ser fermentado.

El bioetanol puede ser usado en forma pura en vehículos especialmente adaptados o mezclado con gasolina. Las mezclas con $5 \%$ o $10 \%$ de etanol en la gasolina se denominan E5 y E10, respectivamente (Sanchez \& Cardona, 2006).

\section{B. BIODIESEL}

El biodiesel es un combustible no derivado del petróleo que consiste en esteres que se pueden obtener tanto de la transesterificación de los triglicéridos, presentes en los aceites vegetales, como de la esterificación de los ácidos grasos libres (Dufey, 2006; Machado, 2010). Sus propiedades son similares a las del diésel convencional. Al ser un combustible obtenido de fuentes naturales tiene características que hacen su uso favorable al medio ambiente. Las características finales del biodiesel dependen de la materia prima utilizada para su procesamiento.

La transesterificación consiste en mezclar aceite o grasa con un alcohol en presencia de un catalizador, provocando una reacción en la que se genera como producto principal alquiléster (biodiesel) y como subproducto un trialcohol (glicerina). La transesterificación es la vía de producción de biodiesel más común (Fukuda, Kondo \& Noda, 2001). En la primera generación, el aceite se produce a partir de oleaginosas o frutos de árboles tales como girasol, soya, palma y cocotero. Existe una segunda generación de biodiesel obtenido de grasas animales, cebo, algas y aceite de cocina usado. En esta generación también se produce biodiesel a partir de madera o paja (Cepeda \& Ochoa, 2004). Sin embargo, para la extracción de los aceites en esta generación, la materia prima debe someterse previamente a procesos de conversión bioquímicos.
De forma similar al bioetanol, el biodiesel se puede usar de forma pura en vehículos especialmente adaptados o mezclado con diésel para automóviles. Por ejemplo, una mezcla de 5\% de biodiesel en diésel fósil se denomina B5 (Dufey, 2006; Sanchez \& Cardona, 2006).

En la tercera generación, la materia prima es común para el bioetanol y el biodiesel: micro algas. No obstante los procesos de obtención son diferentes. Para el biodiesel, el aceite extraído de las microalgas es mezclado con alcohol y un ácido o una base para producir metilésteres de ácido graso (transesterificación). Para el bioetanol, el residuo que queda de las microalgas después de extraerle el aceite, debido a su alto contenido de almidón, es convertido en azucares. Luego, estos azúcares son fermentados por medio de levaduras para la obtención del alcohol.

\section{BIOCOMBUSTIBLES EN COLOMBIA}

Colombia es un país biodiverso, con tierras subutilizadas (Rodríguez, 2000), que pueden usarse para nuevas plantaciones y diversidad de climas necesarios para la proliferación de cultivos. Esto lo convierte en un productor potencial de biocombustibles (Zapata, Franco \& Dyner, 2011).

\section{A. LEGISLACIÓN}

Las legislaciones de la mayoría de los países permiten utilizar etanol mezclado con gasolina en concentraciones del $5 \%$ y $10 \%$ de etanol, que no requieren modificaciones en los motores actuales. Concentraciones más elevadas, implican que se debe disponer de un vehículo flexible, con un depósito, motor y sistema de combustible capaz de funcionar con gasolina y etanol, solos o mezclados en cualquier proporción. Por otra parte, el etanol e isobuteno por reacción catalítica se utiliza en forma de aditivo de la gasolina como etil-terbutiléter (ETBE).

La aprobación de la ley 693 del 2001 (Ministerio de Minas y Energía, 2001) marcó la entrada de Colombia en la nueva era de los biocombustibles. Esta ley fue motivada por el protocolo de Kyoto y la alta dinámica del precio del petróleo (Herrera \& otros, 2009). En esta ley se definen los incentivos que se otorgan para la producción de biocombustibles y se establece el porcentaje de mezcla en ciudades con más de 500000 habitantes (Zapata, Franco \& Dyner, 2011). 
Algunos de los incentivos más representativos de esta ley son (Zapata, Franco \& Dyner, 2011):

- Exclusión del IVA a la caña de azúcar.

- Exención de la renta a la palma de aceite.

- Control de los precios de venta del bioetanol y el biodiesel.

Según el decreto 1135 del 31 de marzo de 2009, era obligatorio que en el año 2012 el $60 \%$ de los vehículos de hasta 2lts vendidos en el país fueran Flex-Fuel E85, es decir, que funcionaran con una mezcla de $85 \%$ de etanol y $15 \%$ de gasolina. Sin embargo, el porcentaje de mezcla tanto para el biodiesel como para el bioetanol descendió, debido a la imposibilidad de satisfacer la demanda local, por la insuficiente capacidad instalada. Posteriormente, para el año 2011 con el decreto 4892, por el cual se dictaron disposiciones aplicables al uso de alcoholes carburantes y biocombustibles para vehículos automotores, se fijó una mezcla del $8 \%-10 \%$ para el bioetanol y del $10 \%$ para el biodiesel. En lo corrido del año, el Gobierno estudia la posibilidad de aumentar el porcentaje de mezcla de biocombustibles de acuerdo al incremento en la producción, además de considerar la posibilidad de crear una segunda mezcla entre $25 \%$ y 85\% para tecnología Flex Fuel.

En general, la regulación de este subsector ha sido dinámica y se ha venido adaptando a las circunstancias internas y externas, con el fin de obtener un desarrollo coherente de la industria de los biocombustibles en el país (Herrera \& otros, 2009).

\section{B. PRODUCCIÓN DE BIOCOMBUSTIBLES}

En Colombia para la producción a gran escala de bioetanol se utiliza como materia prima la caña de azúcar. Mientras que para la producción de biodiesel se utiliza el aceite de palma africana. La capacidad de las plantas productoras de biocombustibles en el país se presenta en las Tablas 1 y 2, para el bioetanol y el biodiesel, respectivamente. La mayoría de cultivos de caña dedicados a la obtención de bioetanol se encuentran en el valle geográfico del Río Cauca, como se observa en la Tabla 1.

Tabla 1. Plantas productoras de bioetanol

\begin{tabular}{llr}
\multicolumn{1}{c}{$\begin{array}{c}\text { Planta de } \\
\text { Refinación }\end{array}$} & \multicolumn{1}{c}{ Ubicación } & $\begin{array}{c}\text { Capacidad } \\
\text { (L/día) }\end{array}$ \\
Incauca & Cauca, Miranda & 350.000 \\
\hline Providencia & Valle, Palmira & 300.000 \\
Manuelita & Valle, Palmira & 250.000 \\
Mayagüez & Valle, Candelaria & 250.000 \\
Risaralda & Risaralda, La Virginia & 100.000
\end{tabular}

Fuente: Ministerio de Minas y Energía, 2013
Tabla 2. Plantas productoras de biodiesel.

\begin{tabular}{|c|c|c|}
\hline $\begin{array}{l}\text { Planta de } \\
\text { Refinación }\end{array}$ & Ubicación & $\begin{array}{l}\text { Capacidad } \\
\text { (L/día) }\end{array}$ \\
\hline Oleoflores & Cesar, Codazzi & 60.000 \\
\hline Odin Energy & $\begin{array}{l}\text { Magdalena, Santa } \\
\text { Marta }\end{array}$ & 36.000 \\
\hline $\begin{array}{l}\text { Biocombustibles } \\
\text { sostenibles del } \\
\text { Caribe }\end{array}$ & $\begin{array}{l}\text { Magdalena, Santa } \\
\text { Marta }\end{array}$ & 100.000 \\
\hline BioD & $\begin{array}{l}\text { Cundinamarca, } \\
\text { Facatativá }\end{array}$ & 115.000 \\
\hline $\begin{array}{l}\text { Ecodiesel de } \\
\text { Colombia S.A. }\end{array}$ & $\begin{array}{l}\text { Santander, } \\
\text { Barrancabermeja }\end{array}$ & 115.000 \\
\hline $\begin{array}{l}\text { Aceites Manuelita } \\
\text { S.A. }\end{array}$ & $\begin{array}{l}\text { Meta, San Carlos de } \\
\text { Guarona }\end{array}$ & 120.000 \\
\hline Biocastilla & Castilla la grande, Meta & 15.000 \\
\hline
\end{tabular}

Fuente: Ministerio de Minas y Energía, 2013

\section{Producción de bioetanol}

Colombia por su ubicación geográfica permite el cultivo y cosecha de materias primas a lo largo de todo el año. Hasta ahora, el bioetanol producido en Colombia proviene casi exclusivamente del procesamiento de la caña de azúcar del Valle de los Ríos Cauca y Meta. Por sus condiciones agroclimáticas ideales: brillo solar permanente e intenso a lo largo del año, balance adecuado de la temperatura entre el día y la noche, disponibilidad de agua, lluvias proporcionadas y suelos fértiles; estas regiones se sitúan dentro de las mejores regiones para cultivar caña del mundo (Herrera \& otros, 2009; Plantas de biodiesel y etanol en Colombia, 2011).

\section{Producción de biodiesel}

La palma de aceite demanda tierra, agua y clima exigentes, además de altos costos de producción (Zapata, Franco \& Dyner, 2011). No obstante, es el cultivo oleaginoso que mayor cantidad de aceite produce por unidad de área sembrada y por lo tanto, es la materia prima que ofrece mejores posibilidades para su producción a nivel nacional (Zapata, Franco \& Dyner, 2011; Herrera \& otros, 2009; Pérez, 2010).

En Colombia, la producción industrial de biodiesel inició en enero de 2008 y se optó por la utilización del aceite de palma como materia prima, dados los desarrollos alcanzados en este sector. En este sentido, Colombia es el mayor productor de aceite de palma en Latinoamérica y el quinto en el mundo (Plantas de biodiesel y etanol en Colombia, 2011). 
En general, entre el $70 \%$ y el $90 \%$ del costo de producción del biodiesel está asociado al costo de la materia prima, según las investigaciones efectuadas por la UPME (Herrera \& otros, 2009). Es por esto que materias primas como la Jatropha, la Higuerilla y el aceite de cocina, por su bajo costo de obtención, se vislumbran como insumos tentativos para la producción del biodiesel (Herrera \& otros, 2009).

\section{IMPACTO EN LOS MOTORES DE COMBUSTIÓN INTERNA}

La conciencia despertada debido a la contaminación atmosférica producto de las emisiones generadas por el sector transporte, ha promovido la búsqueda de alternativas energéticas. Esto ha colocado a los biocombustibles como un tema central de las investigaciones en el área energética.

La incorporación de los biocombustibles en el parque automotor en un país requiere considerar factores como: la disponibilidad, que implica la producción y distribución, la intervención de la legislación, para el establecimiento de la mezcla a implementar, y realizar los cambios necesarios en los motores, entre otros.

En Colombia se promovió el cambio de combustible fósil a mezclas con biocombustibles mediante el modelo Brasilero, sin tener en cuenta las diferencias fundamentales entre el parque automotor de los dos países (Mantilla, 2010; Rosillo-Calle \& Cortez, 1998). El gobierno colombiano se propuso impulsar la producción de biocombustibles buscando primordialmente, la disminución de la dependencia de los combustibles fósiles. La implementación de este programa se proyectó en diferentes fases que incluían el centro del país, al inicio, y que recientemente se ha completado para todo el territorio colombiano.

La mayoría de los autos colombianos a gasolina soportan una mezcla de E10 y B20, sin necesidad de realizar cambios en los vehículos (Mantilla, 2010). El uso de mezclas superiores involucra cambios en los sistemas de alimentación y en algunas partes de los motores, que deben ser considerados. Lo cual, genera costos adicionales al simple hecho de adquirir el nuevo combustible. Estudios realizados por la Universidad Nacional de Colombia (Rosillo-Calle \& Cortez, 1998; Piamba \& Collazos, 2007) ponen en evidencia las partes del sistema de alimentación del combustible, que deberían ser reemplazadas con el propósito de asegurar su estabilidad durante el uso de combustibles más oxigenados.

\section{A. IMPACTO DEL BIOETANOL EN LOS MOTORES (MCI) A GASOLINA}

El bioetanol como combustible tiene un desarrollo de casi 30 años en el mundo (MacLean \& Lave, 2003), existe un panorama difuso sobre las propiedades físico-químicas de las mezclas gasolina-etanol y su comportamiento cuando se utilizan en motores de combustión interna (Goméz, Samaniego \& Antonissen, 2008; Mantilla, 2010). Expertos (Mantilla, 2010; MacLean \& Lave, 2003) coinciden en que no es clara la relación entre las propiedades, el desempeño y las emisiones finales del motor. Esto en gran parte puede deberse a la existencia de calidades variables de gasolina y a la gran variedad de motores empleados en el mundo (Goméz, Samaniego \& Antonissen, 2008). También, a los pocos trabajos que se enfocan en el estudio de la combustión de mezclas gasolina-etanol. Esto último, debido a que los cambios realizados en los motores en países como Brasil y Estados Unidos han correspondido a un largo proceso de prueba y error (MacLean \& Lave, 2003). Sin embargo, algunos autores (Orbital Engine Company, 2002) mencionan problemas asociados a la poca lubricación que ofrece la mezcla de E10 en la cámara de combustión comparada con la gasolina. Otro efecto indeseable es el aumento probable de la corrosión en elementos metálicos del motor debido a la presencia de agua, ya que el etanol es higroscópico (MacLean \& Lave, 2003).

En el proceso de combustión en los motores, es en donde se transforma la energía química de la mezcla en energía mecánica. El proceso de combustión de los biocombustibles trae consigo relaciones de airecombustible menores que aquellas presentes en los procesos de combustión con combustibles derivados del petróleo (Hsieh, 2002). El oxígeno presente en el biocombustible proporciona comburente adicional y la relación aire-combustible debe disminuir.

Los biocombustibles en base alcohol pueden contener una considerable cantidad de agua y, además, se puede producir la oxidación parcial de sus componentes por su exposición a la atmósfera. Estas reacciones ocasionan corrosión de cualquier componente metálico en contacto con las mezclas etanol-gasolina.

Cuando se utilizan biocombustibles en motores de combustión interna, el consumo de estos aumenta (MacLean \& Lave, 2003). Esto se debe a que si se disminuye la relación de aire-combustible estequiométrica para la misma velocidad de operación del motor, el mismo nivel de carga y la misma masa de 
aire, la masa de combustible que se necesita debe ser mayor (Goméz, Samaniego \& Antonissen, 2008).

De otro lado, la potencia (P) y el torque (T) no disminuyen a pesar de que el poder calorífico inferior (LHV) del bioetanol es menor que el de los combustibles fósiles. Inclusive, en algunos casos $\mathrm{P}$ y $\mathrm{T}$ aumentan junto con la eficiencia térmica para las mismas condiciones de operación (Shing, Muttamara \& Lantanakulb, 2002; Hsieh, 2002). Una explicación para este fenómeno es que la temperatura para el autoencendido del bioetanol es mayor que la de la gasolina y el diésel, así como su calor de vaporización y el número de octano (Hansen, Zhang \& Lyne, 2005). Esto revela entonces la posibilidad de tener una temperatura más alta de combustión, dependiendo de varios factores de operación del motor, a medida que el contenido de combustible oxigenado aumenta. Lo cual genera, un posible (ver párrafo siguiente) aumento proporcional en las emisiones de óxidos de nitrógeno (Ocampo, 2006), un deterioro prematuro de los motores al trabajar en condiciones de diferentes a aquellas para las que fue diseñado y daños principalmente en las partes lubricadas. Lo anterior tiene como consecuencia un menor tiempo esperado de vida de los componentes móviles principales del motor, debido a la degradación más rápida del aceite lubricante, i.e., la tasa de oxidación del lubricante aumenta el doble por cada $10^{\circ} \mathrm{C}$ de aumento de temperatura del motor en promedio. Este problema está relacionado directamente con la forma en que se desarrolla el proceso de combustión de mezclas gasolina-etanol en los MCI encendidos por chispa.

Respecto a las emisiones producidas por la combustión, se resalta la reducción en las emisiones de monóxido de carbono (CO) y el aumento del dióxido de carbono $\left(\mathrm{CO}_{2}\right)$ emitido al ambiente, el cual es proporcional a la disminución de $\mathrm{CO}(\mathrm{Wu}, 2004)$. El $\mathrm{CO}_{2}$ producido no tiene efecto directo como gas invernadero, ya que proviene del proceso de combustión de un combustible cuya materia prima es una fuente renovable de energía a partir de biomasa y si se considera el ciclo completo de carbono (Boyle, Everett \& Ramage, 2003). Las emisiones de $\mathrm{NO}_{x}$ están relacionadas directamente con las condiciones de operación del motor, especialmente la temperatura. No obstante, es difícil predecir la tendencia de esta emisión, ya que no existe una relación pre-establecida entre el combustible utilizado y los parámetros mencionados (Boyle, Everett \& Ramage, 2003). El mismo comportamiento variable se presenta para las emisiones de hidrocarburos sin quemar (HC) (Yuksel \& Yuksel, 2004). Estos pueden presentarse en forma de hidrocarburos pesados, procedentes de aceites lubricantes y combustibles derivados de petróleo y también de hidrocarburos livianos en estado gaseoso. En motores con ciclo de presión constante o ciclo de volumen constante, las emisiones de material particulado disminuyen a medida que aumenta la proporción de etanol en la mezcla (Behrentz, 2006). Además, aparecen residuos de etanol sin quemar (posible separación de componentes) en los múltiples de admisión y de escape (Poulopolus, Samaras \& Philippopoulos, 2001; He, 2003).

También es importante resaltar las emisiones no reguladas, como los aldehídos, que aumentan respecto a cuándo se usa gasolina (Yuksel \& Yuksel, 2004). Existen estudios que confirman la probabilidad de desarrollar cáncer y enfermedades relacionadas en seres humanos debido a ellos (Yuksel \& Yuksel, 2004; Ceviz \& Ksel, 2004; Shing, Muttamara \& Lantanakulb, 2002).

La geografía en Colombia y la altura de las ciudades son variadas, por tanto las condiciones de operación de los motores cambian considerablemente desde el nivel del mar hasta ciudades como Tunja o Bogotá con una altura aproximada de 2600 metros sobre el nivel del mar. Otro aspecto importante en Colombia es la variedad del parque automotor. Bogotá es un ejemplo, cuenta con más de un millón de vehículos, de los cuales el $9 \%$ son particulares y el restante son públicos. El promedio de antigüedad de los vehículos particulares es de 15 años y el de los públicos de 25 años (Mantilla, 2010). Por tanto, muchos automotores que circulan por las carreteras colombianas no cuentan con un sistema de control de emisiones apto y utilizan tecnologías de dosificación de combustible obsoletas (Agudelo, Agudelo \& Benjumea, 2007).

En la Tabla 3, se aprecia el resumen comparativo de la influencia en los vehículos entre el bioetanol y la gasolina. 
Tabla 3. Bioetanol (en mezcla) comparado con la gasolina convencional.

\begin{tabular}{|c|c|}
\hline $\begin{array}{l}\text { Parámetro a } \\
\text { comparar }\end{array}$ & Comportamiento \\
\hline $\begin{array}{l}\text { Beneficios de } \\
\text { emisiones }\end{array}$ & $\begin{array}{l}\text { Reduce las emisiones de monóxido } \\
\text { de carbono. Comportamiento variable } \\
\text { en cuanto a los óxidos de Nitrógeno e } \\
\text { inquemados. La emisión de aldehídos } \\
\text { tiende a aumentar }\end{array}$ \\
\hline $\begin{array}{l}\text { Conversión del } \\
\text { motor }\end{array}$ & Necesaria \\
\hline $\begin{array}{l}\text { Ajuste y } \\
\text { regulación del } \\
\text { motor }\end{array}$ & Necesaria \\
\hline Torque & Aumenta \\
\hline Potencia & $\begin{array}{c}\text { Aumenta } \\
(9 \% \text { con una mezcla E20) }\end{array}$ \\
\hline Consumo & $\begin{array}{c}\text { Aumenta } \\
(4 \% \text { para mezclas E15) }\end{array}$ \\
\hline Lubricidad & Disminuye \\
\hline $\begin{array}{l}\text { Respuesta } \\
\text { del motor en } \\
\text { condiciones } \\
\text { invernales }\end{array}$ & Problemático \\
\hline Corrosión & $\begin{array}{l}\text { Aumenta en las partes metálicas y } \\
\text { componentes de caucho }\end{array}$ \\
\hline $\begin{array}{l}\text { Temperatura de } \\
\text { ignición }\end{array}$ & $\begin{array}{c}\text { Disminuye } \\
\text { Bioetanol: } 363^{\circ} \mathrm{C} \\
\text { Gasolina: } 456^{\circ} \mathrm{C}\end{array}$ \\
\hline Emisiones & $\begin{array}{c}\text { Disminuye } \\
\text { Reducción de CO y HC a medida que } \\
\text { se aumenta el porcentaje de alcohol } \\
\text { en la mezcla }\end{array}$ \\
\hline
\end{tabular}

\section{B. IMPACTO DEL BIODIESEL EN LOS MOTORES DE COMPRESIÓN}

En diferentes trabajos encontrados en la literatura, se han determinado las consecuencias del uso del biodiesel en los motores con respecto a las emisiones de gases, la potencia y el consumo (Ganduglia, 2009), la dilución del aceite por acción del combustible y el desgaste del motor (Oil Fox, la marca del biodiesel). En la Tabla 4, se comparan las emisiones de gases contaminantes que genera un motor utilizando B100 y otro con B20, con respecto a las emisiones que produce un motor que usa diésel fósil.

Cabe destacar de la Tabla 4, que al usar B100 los sulfatos se reducen totalmente, el $\mathrm{CO}$ disminuye en un $44 \%$ y los $\mathrm{HC}$ disminuyen en un $68 \%$. Se aprecia que el único efecto negativo es el incremento de $\mathrm{NO}_{x}$. Estos datos son relativos ya que dependen del diseño y tipo del motor (sea para automóviles, vehículos de transporte público, maquinaria pesada, etc.), del tipo de combustible según su composición, la materia de la que proviene, la antigüedad y mantenimiento del motor. Otro aspecto importante es que el humo generado por un vehículo que utiliza biodiesel es menor al momento del arranque en comparación a un vehículo que utiliza diésel (Benavides, Benjumea \& Pashova, 2007). Al utilizar biodiesel existe una pérdida de potencia de hasta un 15\% y un aumento del consumo de combustible, esto debido a que el biodiesel posee un poder energético menor al diésel convencional (121.000BTU y 135.000BTU respectivamente) (Jimenez, 2008). Esto ocurre con mezclas superiores al $20 \%$, en mezclas inferiores, la pérdida de potencia es mínima y el funcionamiento del motor es similar al del diésel convencional.

Tabla 4. Emisiones medidas del biodiesel comparadas al diésel convencional.

\begin{tabular}{|c|c|c|}
\hline Tipo de emisión & $\begin{array}{c}\text { B100 } \\
(\%)\end{array}$ & $\begin{array}{l}\text { B20 } \\
(\%)\end{array}$ \\
\hline $\begin{array}{l}\text { Hidrocarburos totales sin quemar } \\
\text { (HC) }\end{array}$ & -68 & -14 \\
\hline Monóxido de carbono (CO) & -44 & -9 \\
\hline Partículas en suspensión (PM) & -40 & -8 \\
\hline Sulfatos & -100 & -20 \\
\hline $\begin{array}{l}\text { Hidrocarburos aromáticos } \\
\text { policíclicos (PAH) }\end{array}$ & -80 & -13 \\
\hline $\begin{array}{l}\text { Hidrocarburos aromáticos poli } \\
\text { cíclicos nitrogenados (nPAH) }\end{array}$ & -90 & -50 \\
\hline $\begin{array}{l}\text { Potencial de destrucción de la capa } \\
\text { de ozono }\end{array}$ & -50 & -10 \\
\hline Óxidos de nitrógeno $\left(\mathrm{NO}_{\mathrm{x}}\right)$ & +6 & +1 \\
\hline
\end{tabular}

Fuente: Jimenez, 2008

El funcionamiento típico de los motores diésel, permite el paso de una pequeña cantidad de combustible al aceite del motor por medio de los anillos. Esto provoca la reducción de la habilidad lubricante del aceite, el espesamiento del mismo y el desgaste subsecuente del motor. Sin embargo, este comportamiento varía con el uso de biodiesel, debido a su alta lubricidad, que permite la reducción de partículas de metal y carbón en el aceite, aumentando la vida útil del motor, tanto en motores de inyección directa como indirecta.

El biodiesel posee un alto valor de lubricidad aumentando la capacidad de lubricación de los componentes. En la Tabla 5, se muestra la lubricidad de diferentes mezclas de biodiesel a través de los sistemas BOCLE (Ball-on Cylinder Lubricity Evaluator). Se puede apreciar que a medida que aumenta el porcentaje de biodiesel en la mezcla también lo hace la lubricidad. 
Tabla 5. Evaluación BOCLE de lubricidad

\begin{tabular}{|ccc|}
$\begin{array}{c}\text { Porcentaje de } \\
\text { mezcla Diésel- } \\
\text { Biodiesel (\%) }\end{array}$ & $\begin{array}{c}\text { Diésel del petróleo } \\
\text { BOCLE (gramos) }\end{array}$ & $\begin{array}{c}\text { Diésel con bajo } \\
\text { nivel de azufre } \\
\text { BOCLE (gramos) }\end{array}$ \\
\hline 0 & 2200 & 4250 \\
\hline 0,1 & 2750 & 5000 \\
\hline 0,2 & 3450 & 5000 \\
\hline 0,3 & 3200 & 5550 \\
\hline 0,4 & 3500 & 5500 \\
\hline 1 & 3200 & 5700 \\
\hline 10 & 6000 & 6000 \\
\hline 20 & 6000 & 6000 \\
\hline 100 & 6000 & 6000 \\
\hline
\end{tabular}

Fuente: Jimenez, 2008

El uso prolongado de biodiesel tiende a ablandar y degradar ciertos tipos de elastómeros y compuestos de caucho natural usados en mangueras y sistemas de sellado de bombas de combustible. Esto ocurre con los vehículos modelo 1994 y más antiguos. La mayoría de los vehículos construidos luego de 1994 poseen tuberías y sellos completamente sintéticos, por lo cual no tendrán este problema. El efecto solvente que puede liberar depósitos acumulados en las paredes del tanque de combustible o en las tuberías, obstruyendo los filtros del sistema. Se deben tomar precauciones para evitar que estos depósitos lleguen a los filtros de combustible del motor. En una prueba realizada por Instituto de Investigación de Southwest sobre la resistencia a la tensión, elongación, dureza y variaciones de volumen de ciertos elastómeros en presencia B100, B20 y B30, se comprobó que el biodiesel puro o mezclado es compatible con todos los elastómeros probados (Teflón, Nylon 66, Nitrilo, Viton A 401C, Viton GFLT, Fluorsiliconas, Poliuretano, Polipropileno), aunque hubo pequeñas diferencias con la goma de nitrilo. Los elastómeros que mejor desempeño tuvieron con el diésel son el Teflón, Viton A 401C y Viton GFLT. Las casas fabricantes recomiendan que las gomas butílicas o naturales no entren en contacto con biodiesel puro, pues quedarán pegajosas y se disolverán.

Con respecto al almacenaje y el transporte del biodiesel debe tenerse presente la compatibilidad con metales y aleaciones que contengan cobre, bronce, plomo, zinc y estaño pues estos metales pueden catalizar reacciones químicas de degradación del combustible (Methanol
Institute, 2011). Utilizar recipientes de polietileno o polipropileno muy delgado puede provocar problemas de filtraciones, para evitar eso se debe depositar en contenedores de plástico hechos de polipropileno de alta densidad u otros plásticos con un recubrimiento interior fluorado (Methanol Institute, 2011). Los materiales recomendados para el almacenaje de biodiesel son el acero inoxidable, el aluminio, el polipropileno fluorado, el polietileno fluorado, el teflón y la fibra de vidrio (Methanol Institute, 2011).

El biodiesel no es toxico y como se observa en la Figura 1, es degradable. Se degrada entre 4 y 5 veces más rápido que el diésel, por lo que extiende la vida útil de los motores y puede ser usado como solvente para limpiar derrames de diésel fósil.

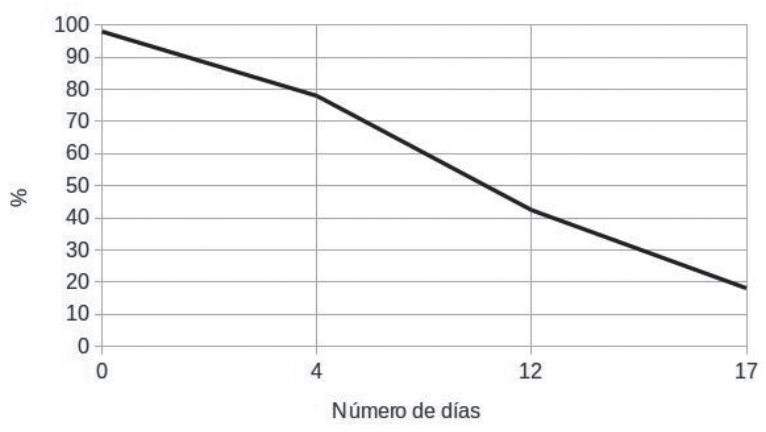

Figura 1. Degradabilidad del biodiesel en función del número de días

Fuente: Fedepalma

En general las precauciones que se toman para el biodiesel son las mismas que para el diésel, minimizar contenido de agua y otros contaminantes, minimizar la exposición del sol y minimizar cambios de temperatura (Bernand, 2006). Particularmente, el biodiesel se oxida si permanece almacenado por periodos de tiempo mayores a seis meses, experimentando cambios en su número ácido, viscosidad y número de cetano (Bernand, 2006). Para tiempos mayores de almacenaje se recomienda usar aditivos especiales que lo estabilicen, como el TBHQ (Butil Hidroquinona Terciario) (Bernand, 2006).

En la Tabla 6, se aprecia el resumen comparativo del efecto del biodiesel en el comportamiento de los motores de compresión. 
Tabla 6. Biodiesel (B20) comparado con el diésel fósil.

\section{Parámetro a comparar}

\begin{tabular}{|c|c|}
\hline Beneficio de emisiones & $\begin{array}{l}\text { Reduce partículas en suspensión, } \\
\text { monóxido de carbono e } \\
\text { hidrocarburos totales }\end{array}$ \\
\hline Conversión del motor & No necesaria \\
\hline $\begin{array}{l}\text { Ajuste y regulación del } \\
\text { motor }\end{array}$ & No necesaria \\
\hline Torque & Similar \\
\hline Potencia & Similar \\
\hline Consumo & Similar \\
\hline Lubricidad & Mayor \\
\hline $\begin{array}{l}\text { Respuesta del motor en } \\
\text { condiciones invernales }\end{array}$ & Similar \\
\hline
\end{tabular}

Seguridad $\begin{gathered}\text { Sin peligro de explosión por } \\ \text { emanaciones }\end{gathered}$
Mayor

Punto de ignición

Biodiesel: $149^{\circ} \mathrm{C}$

Diésel fósil: $67^{\circ} \mathrm{C}$

\begin{tabular}{ll} 
Almacenaje & \multicolumn{1}{c}{ Similar } \\
& Menos agresivas: Para un motor \\
Emanaciones & de $7.3 \mathrm{~L}$, ocurre una variación del \\
& $75 \%$ para $\mathrm{HC}, 1,7 \%$ para NOx y \\
& $33,3 \%$ para CO \\
\hline
\end{tabular}

Fuente: Rodriguez \& Ribeiro, 2009; Bernand, 2006.

\section{LINEAS DE INVESTIGACIÓN ACTUAL ACERCA DE LOS BIOCOMBUSTIBLES}

En la actualidad, la producción de biocombustibles se basa en materias primas que pueden competir con la seguridad alimentaria. Sin embargo, se han venido desarrollando otras generaciones de biocombustibles que permiten la utilización de materias primas consideradas como desecho de actividades agrícolas y otras materias primas como las microalgas. Las cuales, se caracterizan por su capacidad energética y tiempos cortos de reproducción; y las bacterias.

Las microalgas son organismos microscópicos unicelulares que se encuentran de forma natural en el agua dulce y salada. Están ubicadas en la parte inferior de la pirámide alimenticia, lo que aclara por qué no compiten con la seguridad alimentaria (Alam \& Datea, 2012). Existen más de 300000 especies de microalgas, esta diversidad es mucho mayor que la de las plantas (Alam \& Datea, 2012; Scott \& Davey, 2010). Son talofitas, es decir, carecen de raíces, tallos y hojas, tienen clorofila como su pigmento fotosintético primario y carecen de una cubierta estéril alrededor de las células reproductivas (Alam \& Datea, 2012; Brennan \& Owende, 2010). Si bien el mecanismo de la fotosíntesis en estos microorganismos es similar al de las plantas superiores, las microalgas son generalmente más eficientes a la hora de transformar la energía solar gracias a su estructura celular sencilla. Además, debido a que las células crecen en suspensión acuosa, tienen un acceso más eficiente al agua, $\mathrm{CO}_{2}$, y otros nutrientes (Alam \& Datea, 2012; Brennan \& Owende, 2010; Dragone \& Fernandes, 2010)

Los contenidos de aceite de las microalgas en relación a su peso seco varía. Algunas especies pueden tener contenidos de aceite hasta del 80\% (Suali \& Sarbatly, 2012; Liang \& Sarkany, 2010). Existen microalgas que pueden duplicar su biomasa en 24 horas y el tiempo de duplicación (menor) durante su crecimiento es alrededor de 3,5 horas. Lo que hace de las micro algas una fuente renovable ideal para la producción de biocombustibles (Alam \& Datea, 2012; Chisti, 2007).

El contenido de lípidos de las microalgas, específicamente el contenido en triglicéridos (glicerol esterificado con tres ácidos grasos), es básico para la producción del biodiesel (Chisti, 2007; Eichenberger \& Gribi, 1997; Bigogno \& Khozin-Goldberg, 2002). El éxito de la producción de biodesel a partir de las microalgas depende del contenido de triglicéridos y la productividad de la biomasa. Además, las microalgas son ricas en compuestos bioquímicos tales como el ácido docosahexaenoico (DHA) y la astaxantina, que se pueden utilizar en aplicaciones medicinales y han suscitado un creciente interés en la investigación avanzada. Los compuestos químicos de las microalgas también se pueden incorporar en los cosméticos, los aceites de ácidos grasos poli-insaturados, pueden ser añadidos a las fórmulas infantiles y suplementos nutricionales (Chisti, 2007; Spolaore \& Joannis-Cassan, 2006). Por tanto, es deseable aumentar el contenido de lípidos en las microalgas, ya que gran parte de estos compuestos químicos se encuentran dentro de la célula de lípidos. Estas características hacen de la tercera generación de los biocombustibles, obtenidos de las microalgas, una generación superior (Daroch, Geng \& Wang, 2013).

Los biocombustibles provenientes de algas son vistos como una de las soluciones más prometedoras de la crisis energética mundial y el cambio climático para los años venideros. El alto porcentaje de contenido de lípidos, es una ventaja. Sin embargo, el costo de 
producción de biocombustibles con las microalgas como materia prima es elevado. A pesar de esto, no son descartadas ya que requieren menos tierra en comparación con cultivos comerciales como la palma o la jatropha (Suali \& Sarbatly, 2012; Chisti, 2007; Schenk \& Hall, 2008). Hasta el momento, los métodos más eficaces de producción de biocombustibles a partir de estas materias primas son: producción fermentativa de etanol a partir de algas y la síntesis de biodiesel a partir del aceite de algas (transesterificación) (Daroch, Geng \& Wang, 2013).

El verdadero avance sin embargo se espera a partir de: i) la ingeniería metabólica de los organismos fotosintéticos (cianobacterias), puesto que abre nuevas vías en la producción de biocombustibles de algas y promete una importante simplificación del proceso de producción de biocombustibles (Daroch, Geng \& Wang, 2013); ii) en la cuarta generación de los biocombustibles que pretende obtenerlos a partir de dióxido de carbono o alguna otra fuente de carbono, utilizando microorganismos alterados genéticamente.

En general, el futuro de los biocombustibles, apunta al desarrollo de tecnologías que permitan su obtención de una forma más limpia e eficiente, desde su materia prima hasta el producto final. Enfocado al desarrollo de generaciones que no compitan con la seguridad alimentaria, no contaminen las fuentes hídricas y reduzcan realmente las emisiones de gases de efecto invernadero.

\section{CONCLUSIONES}

- En este momento, los biocombustibles no mitigan el alza e inestabilidad en el precio del petróleo. No obstante, son la opción más viable a largo plazo, logrando disminuir la dependencia de los combustibles fósiles.

- Actualmente, Colombia es un país productor de biocombustibles (bioetanol) de primera generación. En Colombia, se está estudiando la utilización de materias primas diferentes a la caña de azúcar y la palma para la producción de biocombustibles. No obstante, su implementación se limita a plantas piloto, que no afectan la producción comercial actual.

- En términos de competitividad, los biocombustibles varían de acuerdo a las materias primas utilizadas, la tecnología empleada, y el mercado en el que se desarrollen.
- El futuro de los biocombustibles depende de la inversión que se haga en investigación y desarrollo por parte de las naciones. Colombia ocupa una posición privilegiada para el desarrollo de los biocombustibles en el mundo.

- El contenido energético del biodiesel es menor que el del diésel (12\% menor en peso, $8 \%$ en volumen) por lo que su consumo es ligeramente mayor.

- Aunque se han realizado varios estudios, aún en Colombia no se conocen claramente las consecuencias del uso de biocombustibles en vehículos, debido a la diversidad geográfica del país y la variedad del parque automotor. Además, las implicaciones del uso de biocombustibles en motores son afectadas por el tipo de motor que se utilice, el lugar donde se realicen las pruebas, la composición del biocombustible utilizado y la antigüedad del motor, entre otros. Lo anterior, justifica la diversidad en los resultados expuestos en los diferentes trabajos de investigación sobre el tema. Por lo tanto, se recomienda hacer una investigación aplicada de la influencia real de los biocombustibles.

\section{REFERENCIAS}

1. Agudelo, A., Agudelo, J., \& Benjumea, P. (2007). Diagnóstico de la combustión de biocombustibles en motores. Revista Facultad de Ingeniería Universidad de Antioquia, 45, 41-53.

2. Alam, F., \& Datea, A. (2012). Biofuel from algae, Is it a viable alternative? Procedia Engineering, 49, 221227.

3. Atlas de la agroenergía y los biocombustibles en las américas: Etanol. (2008). San José, Costa Rica: Instituto Interamericano de Cooperación para la Agricultura.

4. A literature review based assessment on the impacts of a 20\% Ethanol-Gasoline fuel blend on the Australian vehicle fleet. (2002). Australia: Orbital Engine Company.

5. Behrentz, E. (2006). Beneficios ambientales asociados con el uso de combustibles alternativos. Bogotá, Colombia: XIII Conferencia Energética Colombiana.

6. Benavides, A., Benjumea, P., \& Pashova, V. (2007). El biodiesel de aceite de higuerilla como combustible alternativo en motores diésel. Dyna, 74, 141-150. 
7. Bernand, E. (2006). Biodiesel: Los aspectos mecánicos en los vehículos. San José, Costa Rica: Autor.

8. Bigogno, C., \& Khozin-Goldberg, I. (2002). Lipid and fatty acid composition of the green oleaginous alga Parietochloris incisa, the richest plant source of arachidonic acid. Phytochemistry, 60, 497-503.

9. Brennan, L., \& Owende, P. (2010). Biofuels from microalgae - A review of technologies for production, processing, and extractions of biofuels and coproducts. Renewable and Sustainable Energy Reviews, 14, 557577.

10. Boyle, G., Everett, B., \& Ramage, J. (2003). Energy systems and sustainability, power for a sustainable future. Milton Keynes, UK: Oxford.

11. Cepeda, R., \& Ochoa, L. (2004). Obtención de combustibles sintéticos mediante la reacción de Fischer Tropsch, empleando catalizadores de hierro. Revista Colombiana de Química, 33, 2357-3791.

12. Ceviz, A., \& Ksel, Y. (2004). Effect of Ethanolunleaded gasoline blends on cyclic variability and emissions in an SI engine. Applied Thermal Engineering, 25, 917-925.

13. Chisti, Y. (2007). Biodiesel from microalgae. Biotechnology Advances, 25, 294-306.

14. Daroch, M., Geng, S., \& Wang, G. (2013). Recent advances in liquid biofuel production from algal feedstocks. Applied Energy, 102, 1371-1381.

15. Dragone, G., \& Fernandes, B. (2010). Third generation biofuels from microalgae. Current Research, Technology and Education Topics in Applied Microbiology and Microbial Biotechnology, 2, 1355-1366.

16. Dufey, A. (2006). Producción y Comercio de biocombustibles y desarrollo sustentable: Los grandes temas. London, UK: International, Institute for Environment and Development.

17. Eichenberger, W., \& Gribi, C. (1997). Lipids of Pavlova Lutheri: cellular site and metabolic role of DGCC. Phytochemistry, 45, 1561-1567.

18. Fukuda, H., Kondo, A., \& Noda, H. (2001). Biodiesel fuel production by transesterification of oils. Journal of Bioscience and Bioengineering, 92, 405-416.
19. Ganduglia, F. (2009). Manual de biocombustibles. San José, Costa Rica: Instituto Interamericano de Cooperación para la Agricultura (IICA).

20. Goméz, J., Samaniego, J., \& Antonissen, M. (2008). Consideraciones ambientales entorno a los biocombustibles líquidos. CEPAL, 137, 1-52.

21. Hansen, A., Zhang, Q., \& Lyne, P. (2005). EthanolDiesel fuel blends a Review. Bioresource Technology, 96, 277-285.

22. He, B. (2003). A study on emissions characteristics of an EFI engine with ethanol blended gasoline fuels. Atmospheric environment, 37, 949-957.

23. He, B. (2003). The effect of ethanol blended diesel fuels an emissions from a diesel engine. Atmospheric environment, 37, 4965- 4971.

24. Herrera, B., \& otros. (2009). Biocombustibles en Colombia. Bogotá, Colombia: Unidad de Planeación Minero Energética, UPME.

25. Hsieh, W. (2002). Engine performance and pollutant emission of an SI engine using ethanol-gasoline blended fuels. Atmospheric Environment, 36, 403410.

26. Methanol Institute. (2011). Manual de manipulación segura del metanol [En línea]. California, USA: Autor.

27. Jimenez, I. (2008). Transferencia tecnológica sobre las ventajas y desventajas de la utilización de biodiesel. San José, Costa Rica: Instituto Nacional de Aprendizaje.

28. Machado, C.(2010). Situación de los biocombustibles de 2 da y $3 r a$ generación. Brasil: Embrapa.

29. MacLean, H., \& Lave, L. (2003). Evaluating automobile fuel/propulsion system technologies. Progress in energy and combustion science, 29, 1-69.

30. Mantilla, J. (2010). Modelado de la combustión de mezclas gasolina-etanol en motores de combustión interna. Tesis doctoral. Medellín, Colombia: Universidad Nacional de Colombia.

31. Ministerio de Minas y Energía. (2001). Ley 693 de Septiembre 19 de 2001 [En línea]. 
Recuperado de https://www.minminas.gov.co/ documents/10180//23517//21462-3660.pdf.

32. Ministerio de Minasy Energía.(2013).Hidrocarburos [En línea]. Recuperado de https://www.minminas. gov.co/documents/10180/614096/2-Hidrocarburos. pdf/75855d82-def9-4ccb-9fe4-2d4ee97f9123.

33. Liang, Y., \& Sarkany, N. (2010). Use of sweetsorghum juice for lipid production by Schizochytrium limacinum SR21. Bioresour Technol, 101, 36233627.

34. Ocampo, W. (2006). Es la biogasolina una alternativa ambiental en Colombia. Medellín, Colombia: Facultad de Ingeniería, Universidad de Antioquia.

35. Pérez, A. (2010). Biocombustibles en Suramérica: referentes normativos y legislación actual. Derechos $y$ Valores, 13, 215-232.

36. Piamba, O., \& Collazos, O. (2007). Desempeño del motor utilizando como combustible mezcla de etanol anhidro y gasolina en relación de 10\% en Volumen E10. Bogotá, Colombia: Facultad de Ingeniería, Universidad Nacional de Colombia.

37. Poulopolus, S., Samaras, D., \& Philippopoulos, C. (2001). Regulated and unregulated emissions from an internal combustion engine operating on ethanolcontaining fuels. Atmospheric Environment, 35, 4399-4406.

38. Rodríguez, M. (2000). La biodiversidad en Colombia [En línea]. Recuperado de http:/www. manuelrodriguezbecerra.org/bajar/biodiversidad.pdf

39. Rodriguez, G., \& Ribeiro, M. (2009). Estudio comparado entre el combustible diesel y biodiesel. Argentina: Instituto Nacional de Tecnología Industrial.

40. Rosillo-Calle, F., \& Cortez, L. (1998). Towards Proalcool lida Reviews of the Brazilian bioetanol. Programme biomass and bioenergy, 14, 115-124.
41. Sanchez, O., \& Cardona, C. (2006). Producción de alcohol carburante, una alternativa para el desarrollo agroindustrial. Manizales, Colombia: Universidad Nacional de Colombia.

42. Schenk, P., \& Hall, T. (2008). Second generation biofuels: high-efficiency microalgae for biodiesel production. Bioenergy, 1, 20-43.

43. Scott, S., \& Davey, M. (2010). Biodiesel from algae: challenges and prospects. Current Opinion in Biotechnology, 21, 277-286.

44. Shing, L., Muttamara, S., \& Lantanakulb, P. (2002). Aplicability of gasoline containing ethanol as Thailands alternative fuel to curb toxic VOC pollutants from automobile emission. Atmospheric Environment, 36, 3495-3503.

45. Spolaore, P., \& Joannis-Cassan, C. (2006). Commercial application of microalgae. $J$ Biosci Bioeng, 101, 87-96.

46. Stratta, J. (2000). Biocombustibles: Los aceites vegetales como constituyentes principales del biodiesel. Argentina: Bolsa de Comercio de Rosario.

47. Suali, E., \& Sarbatly, R. (2012). Conversion of microalgae to biofuel. Renewable and Sustainable Energy Reviews, 16, 4316-4342.

48. Wu, C. (2004). The influence or air-fuel ratio on engine performance and pollutant emission of an SI engine using ethanol-gasoline blended fuels. Atmosfheric Environment, 38, 7093-7100.

49. Yuksel, F., \& Yuksel, B. (2004). The uses of ethanolgasoline blend as a fuel in an SI engine. Renewable energy, 29, 1181-1191.

50.Zapata, S., Franco, C., \& Dyner, I. (2011). Una aproximación desde la dinámica de sistemas a la liberación del mercado de los biocombustibles en Colombia. Bogotá, Colombia: Universidad Colegio Mayor de Nuestra Señora del Rosario. 